\title{
Condition for gapless color-antitriplet excitations in Nambu-Jona-Lasinio models
}

\author{
F. Sandin ${ }^{1}$ and A. M. Öztaş ${ }^{2}$ \\ ${ }^{1}$ Department of Physics, Luleå University of Technology, SE-97187 Luleå, Sweden \\ ${ }^{2}$ Department of Physics, Hacettepe University, TR-06532 Ankara, Turkey
}

(Received 8 December 2005; published 8 March 2006)

\begin{abstract}
We present an exact condition for the existence of gapless quasiparticle excitations in Nambu-Jona-Lasinio models of color superconducting quark matter with a quark-quark interaction in the scalar color-antitriplet channel. The condition can be represented by a rotated ellipse in the plane of mass and chemical potential differences for the paired quark fields.
\end{abstract}

DOI: 10.1103/PhysRevC.73.035203

PACS number(s): 24.85.+p, 12.38.Mh, 25.75.Nq, 26.60.+c

\section{INTRODUCTION}

At high baryon density and low temperature, matter is believed to be in a color superconducting state, which is characterized by condensates of quark Cooper pairs [1-4]. A superconducting phase typically has an energy gap in the density of states, which corresponds to the lowest excitation energy of a quasiparticle pair. However, if the difference between the Fermi momenta of the paired quarks is sufficiently large, gapless quasiparticle excitations could exist [5-8]. The presence of gapless phases could have observable consequences, e.g., the high specific heat and neutrino emissivity could affect the cooling behavior of compact stars [9]. It has been found, however, that gapless phases might suffer from a chromomagnetic instability [10-15], and it is currently unclear whether gapless phases appear at temperatures relevant for compact star evolution [8,15-17]. It is therefore important to improve the understanding of gapless phases. In this paper we derive an exact condition for the existence of gapless excitations in the frequently used Nambu-Jona-Lasinio (NJL) model of color superconducting quark matter. A qualitatively useful graphical representation of the condition and some well-known approximations are also presented.

\section{MODEL}

The most dense environment where quark matter is expected to exist is in the core of neutron stars, which are subject to a gravitational instability that limits the maximum density to $\sim 10^{15} \mathrm{~g} / \mathrm{cm}^{3}$ [18]. This corresponds to a maximum quarknumber chemical potential of $\mu \sim 500 \mathrm{MeV}$ and a maximum baryon number density of $n_{B} \sim 10 n_{0}$, where $n_{0}=0.17 \mathrm{fm}^{-3}$ is the baryon number density in nuclear matter. Since the charm quark mass is higher than the maximum chemical potential, it is sufficient to consider up $(u)$, down $(d)$, and strange $(s)$ quarks. The quark spinors are

$$
q^{T}=\left(\psi_{u r}, \psi_{u g}, \psi_{u b}, \psi_{d r}, \psi_{d g}, \psi_{d b}, \psi_{s r}, \psi_{s g}, \psi_{s b}\right)
$$

where $r, g$, and $b$ represent red, green, and blue colors. The NJL model of superconducting quark matter is based on effective pointlike four-fermion interactions and is described in, e.g., Refs. [15-17,19]. Here we repeat some of the essential points.
The Lagrangian density is

$$
\mathcal{L}_{\text {eff }}=\bar{q}\left(i \not \partial-\hat{m}+\hat{\mu} \gamma^{0}\right) q+\mathcal{L}_{\bar{q} q}+\mathcal{L}_{q q},
$$

where $\hat{m}=\operatorname{diag}_{f}\left(m_{u}, m_{d}, m_{s}\right)$ is the current quark mass matrix in flavor space. $\mathcal{L}_{\bar{q} q}$ and $\mathcal{L}_{q q}$ are the effective interaction terms, which are used at mean-field level in the Hartree approximation. Explicitly,

$$
\begin{aligned}
\mathcal{L}_{\bar{q} q}= & G_{S} \sum_{a=0}^{8}\left[\left(\bar{q} \tau_{a} q\right)^{2}+\left(\bar{q} i \gamma_{5} \tau_{a} q\right)^{2}\right] \\
& -K\left\{\operatorname{det}_{f}\left[\bar{q}\left(1+\gamma_{5}\right) q\right]+\operatorname{det}_{f}\left[\bar{q}\left(1-\gamma_{5}\right) q\right]\right\}, \\
\mathcal{L}_{q q}= & G_{D} \sum_{a, b=2,5,7}\left(\bar{q} i \gamma_{5} \tau_{a} \lambda_{b} C \bar{q}^{T}\right)\left(q^{T} C i \gamma_{5} \tau_{a} \lambda_{b} q\right),
\end{aligned}
$$

where $\tau_{a}$ and $\lambda_{b}$ are the antisymmetric Gell-Mann matrices acting in, respectively, flavor and color space. $G_{S}, K$, and $G_{D}$ are coupling constants that must be determined by experiments.

The quark-quark interaction term $\mathcal{L}_{q q}$ gives rise to superconducting condensates, $s_{a b}=\left\langle q^{T} C \gamma_{5} \tau_{a} \lambda_{b} q\right\rangle$, which break $\mathrm{SU}(3)_{c}$ and $\mathrm{U}(1)$ symmetry. The symmetries of $\mathcal{L}$ correspond to a conserved chromoelectromagnetic charge. The associated chemical potential is [19]

$$
\hat{\mu}=\mu+\mu_{Q}\left(\frac{\tau_{3}}{2}+\frac{\tau_{8}}{2 \sqrt{3}}\right)+\mu_{3} \lambda_{3}+\mu_{8} \lambda_{8} .
$$

Here, $\mu$ is the quark-number chemical potential, $\mu_{Q}$ is the positive electric-charge chemical potential, and $\mu_{3}$ and $\mu_{8}$ are color-charge chemical potentials. By linearizing Eq. (2) in the quark-quark (diquark) gaps, $\Delta_{a b}=2 G_{D} s_{a b}$, and the quarkantiquark (chiral) gaps, $\phi_{i}=-4 G_{S}\left\langle\bar{q}_{i} q_{i}\right\rangle$, one can obtain a grand canonical thermodynamic potential by standard methods [15-17,19]:

$$
\begin{aligned}
\Omega(T, \mu)= & \frac{\phi_{u}^{2}+\phi_{d}^{2}+\phi_{s}^{2}}{8 G_{S}}+\frac{K \phi_{u} \phi_{d} \phi_{s}}{16 G_{S}^{3}}+\frac{\Delta_{u d}^{2}+\Delta_{u s}^{2}+\Delta_{d s}^{2}}{4 G_{D}} \\
& -\int \frac{d^{3} p}{(2 \pi)^{3}} \sum_{n=1}^{18}\left[E_{n}+2 T \ln \left(1+e^{-E_{n} / T}\right)\right] \\
& +\Omega_{\text {lep }}-\Omega_{0} .
\end{aligned}
$$

Here, $E_{n}\left(p, \mu ; \mu_{Q}, \mu_{3}, \mu_{8}, \phi_{u}, \phi_{d}, \phi_{s}, \Delta_{u d}, \Delta_{u s}, \Delta_{d s}\right)$ are the quasiparticle dispersion relations, $\Omega_{\text {lep }}$ is the contribution from leptons (e.g., electrons, muons, and the corresponding 
neutrino flavors), and $\Omega_{0}$ is the vacuum, i.e., $\Omega(0,0)=0$. It should be noted that Eq. (6) is an even function of $E_{n}$, so the signs of the dispersion relations are arbitrary. We therefore follow the standard convention that all states below the Fermi surface $\left(E_{n}<0\right)$ are occupied, and only positive-energy states are considered. In Eq. (6) the diquark gaps are denoted with flavor indices. One can readily do this by considering the color and flavor structure of the Gell-Mann matrices

$$
\begin{array}{ll}
\Delta_{u d} \equiv \Delta_{22} & (u-d, r-g \text { pairing }), \\
\Delta_{u s} \equiv \Delta_{55} & (u-s, r-b \text { pairing }), \\
\Delta_{d s} \equiv \Delta_{77} & (d-s, g-b \text { pairing })
\end{array}
$$

and $\Delta_{a b}=0$ if $a \neq b$ [19]. The chiral gaps and the diquark gaps are variational parameters that are determined by minimization of Eq. (6). The constituent quark masses are

$$
M_{i}=m_{i}+\phi_{i}+\frac{K}{8 G_{S}^{2}} \phi_{j} \phi_{k},
$$

where $(i, j, k)$ is any permutation of $(u, d, s)$.

In QCD, a color superconducting ground state is automatically color neutral because of the generation of gluon condensates in one or more of the eight components of the gluon field. In NJL models there are no gauge fields that neutralize the color charge dynamically, because the gluons have been replaced with effective pointlike quarkantiquark [Eq. (3)] and quark-quark [Eq. (4)] interactions. One must therefore enforce color neutrality by solving for the charge chemical potentials $\mu_{Q}, \mu_{3}$, and $\mu_{8}$ such that the corresponding charge densities $n_{a}=\left\langle\psi^{\dagger} T_{a} \psi\right\rangle=-\partial \Omega / \partial \mu_{a}$ are zero [20].

The values of the gaps and the (charge) chemical potentials depend on the coupling constants $\left(G_{S}, K\right.$, and $\left.G_{D}\right)$, the current quark masses $\left(m_{u}, m_{d}\right.$, and $\left.m_{s}\right)$, and the regularization method. These input parameters are fitted to low-density hadronic results and are therefore only approximately known. In addition, approximations are frequently used to simplify the evaluation of Eq. (6). In this context it would be useful to have a mathematically exact condition for the appearance of gapless quasiparticle dispersion relations, without reference to specific input parameters and further assumptions. This condition is presented below.

\section{GAPLESS CONDITIONS}

The dispersion relations $E_{n}$ are eigenvalues of six $4 \times 4$ matrices and one $12 \times 12$ matrix [15-17]. Disregarding the signs, three $4 \times 4$ matrices and 6 of the 12 eigenvalues of the $12 \times 12$ matrix remain $(3 \times 4+6=18)$. The $12 \times 12$ matrix corresponds to $u r-d g-s b$ pairing, and the three $4 \times 4$ matrices correspond to $u g-d r, u b-s r$, and $d b-s g$ pairing. There are strong indications that the $u r-d g-s b$ modes are never gapless, because the Fermi momenta of these three species are approximately equal [8], and no such gapless modes have been found in numerical evaluations [15-17]. A proof has turned out to be difficult to obtain because of the complexity of the characteristic polynomial of the $12 \times 12$ matrix. We therefore leave this analysis to a future publication. Here the $4 \times 4$ matrices are considered. The characteristic polynomials of these matrices can be written as

$$
E_{n}^{4}+a_{3} E_{n}^{3}+a_{2} E_{n}^{2}+a_{1} E_{n}+a_{0} .
$$

The $a_{0}$ coefficient of the polynomial is [17]

$$
\begin{aligned}
a_{0}= & p^{4}+\left(M_{i}^{2}+M_{j}^{2}+2 \Delta_{i j}^{2}-\mu_{i \alpha}^{2}-\mu_{j \beta}^{2}\right) \\
& +p^{2}\left(\mu_{i \alpha} \mu_{j \beta}+M_{i} M_{j}+\Delta_{i j}^{2}+\mu_{i \alpha} M_{j}+\mu_{j \beta} M_{i}\right) \\
& \times\left(\mu_{i \alpha} \mu_{j \beta}+M_{i} M_{j}+\Delta_{i j}^{2}-\mu_{i \alpha} M_{j}-\mu_{j \beta} M_{i}\right),
\end{aligned}
$$

for quark flavors $(i, j)$ and colors $(\alpha, \beta)$. The chemical potential $\mu_{i \alpha}$ for a quark field with flavor $i$ and color $\alpha$ can be extracted from Eq. (5) ( $\hat{\mu}$ is diagonal in color and flavor space). A gapless dispersion relation is characterized by $E_{n}(p)=0$ for some real value(s) of $p$ when $\Delta_{i j} \neq 0$. This requires that $a_{0}(p)=0$ have at least one real root. The solutions are

$$
\begin{aligned}
p^{2}= & \bar{\mu}^{2}+\delta \mu^{2}-\bar{M}^{2}-\delta M^{2}-\Delta^{2} \\
& \pm 2 \sqrt{(\bar{\mu} \delta \mu-\bar{M} \delta M)^{2}-\Delta^{2}\left(\bar{\mu}^{2}-\delta M^{2}\right)} .
\end{aligned}
$$

Here we have introduced the quantities

$$
\begin{aligned}
\bar{M} & =\left(M_{i}+M_{j}\right) / 2, \quad \delta M=\left(M_{i}-M_{j}\right) / 2, \\
\bar{\mu} & =\left(\mu_{i \alpha}+\mu_{j \beta}\right) / 2, \quad \delta \mu=\left(\mu_{i \alpha}-\mu_{j \beta}\right) / 2,
\end{aligned}
$$

and $\Delta=\Delta_{i j}$. The indices in Eq. (12) can be omitted without ambiguity, since we are dealing with two-species pairing. Observe that the masses and chemical potentials of the paired quark fields are $\bar{M} \pm \delta M$ and $\bar{\mu} \pm \delta \mu$. A real square root in Eq. (13) requires that

$$
\Delta \leqslant \Delta^{g} \equiv \frac{|\bar{\mu} \delta \mu-\bar{M} \delta M|}{\sqrt{\bar{\mu}^{2}-\delta M^{2}}},
$$

and a positive solution for $p^{2}$ requires that

$$
\begin{aligned}
\bar{M}^{2}+ & \delta M^{2}+\Delta^{2}-\bar{\mu}^{2}-\delta \mu^{2} \\
& \leqslant 2 \sqrt{(\bar{\mu} \delta \mu-\bar{M} \delta M)^{2}-\Delta^{2}\left(\bar{\mu}^{2}-\delta M^{2}\right) .}
\end{aligned}
$$

Inequality (16) can be represented with a rotated ellipse in the $\delta M-\delta \mu$ plane, as in Fig. 1. The interior region of the ellipse violates inequality (16) and hence represents gapped modes. Outside the ellipse the square root in inequality (17) is real, and inequality (17) is obviously satisfied as long as the left-hand side is negative. A negative left-hand side of inequality (17) is represented by the region in between the two branches of the hyperbola, $\bar{\mu}^{2}+\delta \mu^{2}=\bar{M}^{2}+\delta M^{2}+\Delta^{2}$, in Fig. 1. For a positive left-hand side, which corresponds to the two regions on the left- and right-hand sides of the hyperbola, inequality (17) can be squared, and four coupled inequalities linear in $\delta M$ and $\delta \mu$ are obtained. These correspond to tangent lines of the ellipse. The hatched areas enclosed by the tangent lines, the hyperbola and the ellipse violate inequality (17) and hence represent gapped modes. For each tangent line the intersection with the hyperbola coincides with the point on the ellipse. Inequality (17) is relevant if $\delta M / \bar{\mu} \sim 1-\Delta^{2}(\bar{\mu}-\bar{M})^{-2} / 2$, which is not the case for realistic values of the masses and chemical potentials. This is explicitely demonstrated by the examples in Fig. 2. Inequality (16) is therefore the relevant condition for gapless modes. In achieving this result, no further approximations other than those leading up to Eq. (6) were made. 


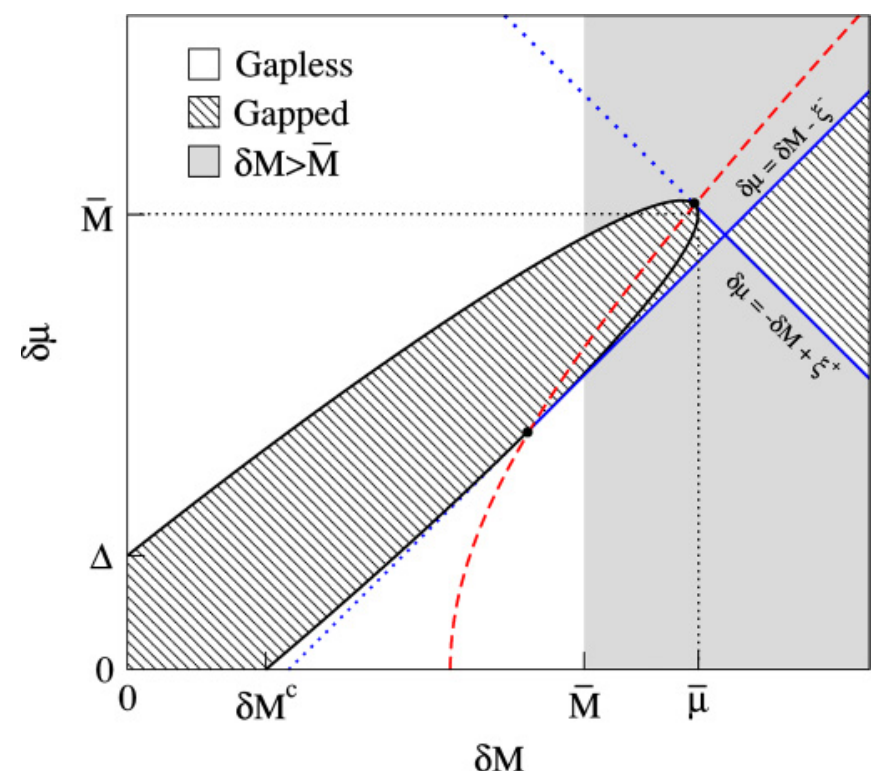

FIG. 1. (Color online) Graphical representation of gapless inequalities (16) and (17). For clarity only the first quadrant is shown. The third quadrant is a reflection of the first quadrant in the origin. In this figure an unreasonably large value of $\bar{M}$ has been used to emphasize the role of the tangent lines. Qualitatively, the gapped region can be represented by the rotated ellipse; see the text. The values of $\delta \mu$ and $\delta M$ can be represented by a point in the $\delta M-\delta \mu$ plane. If this point is enclosed by the hatched area, the dispersion relations are gapped. Otherwise a gapless dispersion relation exists. Here $\delta M^{c}=\bar{\mu} /\left(1+\bar{M}^{2} / \Delta^{2}\right)^{1 / 2}$ and $\xi^{ \pm}=\left[\Delta^{2}+(\bar{\mu} \pm \bar{M})^{2}\right]^{1 / 2}$.

For the two-flavor color superconducting phase, which is characterised by $\Delta_{u d} \neq 0$ and $\Delta_{u s}=\Delta_{d s}=\mu_{3}=0$, one can use the fact that $\delta M \ll \delta \mu$ and $M \ll \mu$, so the gapless

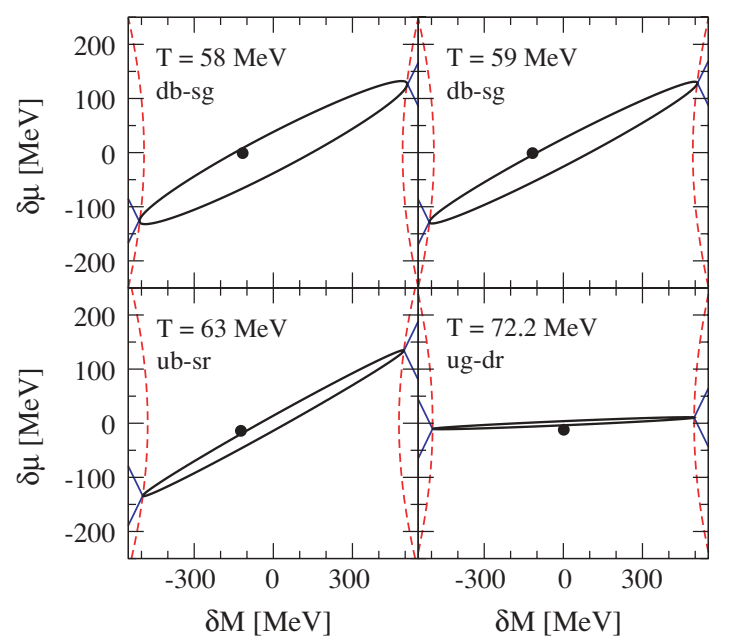

FIG. 2. (Color online) Graphical representation of gapless conditions (16) and (17) for some of the quasiparticle dispersion relations represented in Fig. 4. The values of $\delta M$ and $\delta \mu$ are represented by bold points. If the center of a point is enclosed by an ellipse, the corresponding quasiparticle has gapped dispersion relations; otherwise a gapless dispersion relation exists.

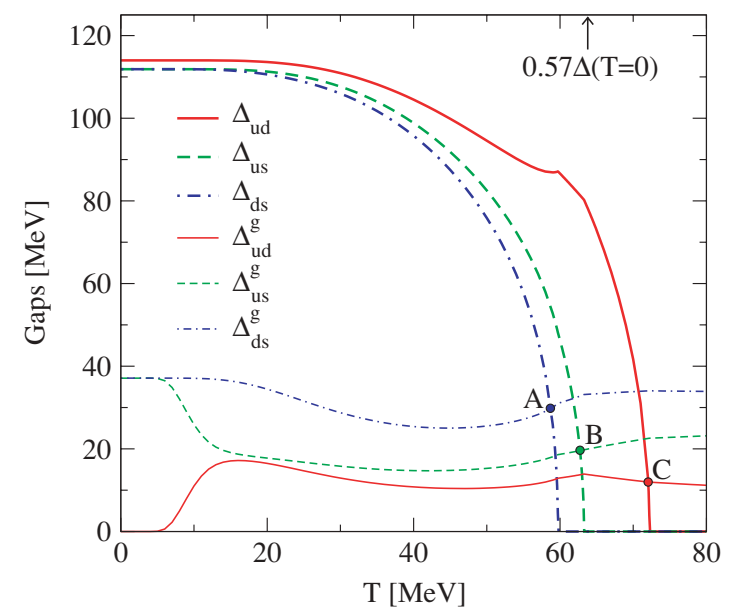

FIG. 3. (Color online) Diquark gaps vs. the temperature at $\mu=$ $500 \mathrm{MeV}$ and $\eta=0.75 . \Delta_{i j}^{g}$ is the threshold for gapless quasiparticle dispersion relations (16), i.e., gapless modes exist iff $\Delta_{i j} \leqslant \Delta_{i j}^{g}$. The critical points where gapless $d b-s g, u b-s r$, and $u g-d r$ quasiparticles appear are denoted by, respectively, $A, B$, and $C$. The BCS result for the critical temperature of a superconducting condensate, $T \sim$ $0.57 \Delta(T=0)$, is indicated in the plot. This figure represents a cross section of Fig. 5 in Ref. [17].

condition [Eq. (16)] is approximately

$$
\Delta_{u d} \lesssim|\delta \mu|=-\mu_{Q} .
$$

For the three-flavor color-flavor-locked phase, which is characterized by $\Delta_{i j} \neq 0$ and $\bar{M} \sim \delta M \sim M_{S} / 2$, a series expansion of inequality (16) to first order in $M_{s}^{2} / \bar{\mu}$ yields

$$
\Delta_{i s} \lesssim|\delta \mu|+\left[\frac{\operatorname{sign}(\delta \mu)}{4}+\frac{|\delta \mu|}{8 \bar{\mu}}\right] \frac{M_{s}^{2}}{\bar{\mu}},
$$

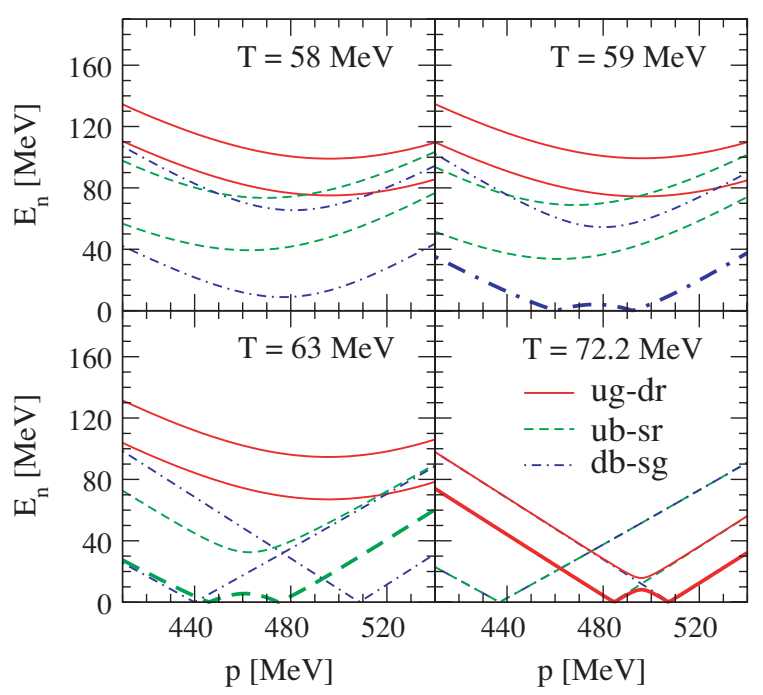

FIG. 4. (Color online) Dispersion relations at $\mu=500 \mathrm{MeV}$ and $\eta=0.75$ for four different temperatures. Gapless modes are denoted by the thick curves. Gapped modes and modes of unpaired quarks are denoted by the thin curves. Compare with Fig. 3. 
where $\delta \mu=\left(\mu_{i \alpha}-\mu_{s \beta}\right) / 2$. These well-known approximate results are instructive at the qualitative level, but should not be used mechanically; see the discussion below.

\section{NUMERICAL EXAMPLE}

Next we present a numerical example and therefore constrain the discussion to a specific parametrization of the model, as in Ref. [17]. The momentum integral is regularized with a cutoff, $\Lambda=602.3 \mathrm{MeV}$. The coupling constants are $G_{S} \Lambda^{2}=$ $2.319, G_{D} / G_{S} \equiv \eta=0.75$, and $K=0$. The current quark masses are $m_{u}=m_{d}=5.5 \mathrm{MeV}$ and $m_{s}=112 \mathrm{MeV}$. By the insertion of these parameters into the thermodynamic potential (6), the gaps $\left(\phi_{u}, \phi_{d}, \phi_{s}, \Delta_{u d}, \Delta_{u s}, \Delta_{d s}\right)$ can be determined by minimization of the free energy, while simultaneously neutralizing all charge densities with $\mu_{Q}, \mu_{3}$, and $\mu_{8}$. In Fig. 3 the diquark gaps $\Delta_{i j}$ and the gapless thresholds [inequality (16)], $\Delta_{i j}^{g}$ are plotted versus the temperature for a fixed value of the quark-number chemical potential, $\mu=$ $500 \mathrm{MeV}$. In Fig. 4 the quark-quark quasiparticle dispersion relations are plotted for four different temperatures represented in Fig. 3. Observe that gapless dispersion relations exist iff $\Delta_{i j} \leqslant \Delta_{i j}^{g}$. Figure 2 shows the graphical representation of the gapless condition for some quasiparticles represented in Fig. 4, see Fig. 1 for further information.

\section{CONCLUSIONS}

We find that the difference between approximate results (18) and (19) and exact gapless condition (16) is typically below 5\% in the plane of temperature and quark-number chemical potential. However, even a small error in $\Delta_{i j}^{g}$ could lead to qualitatively incorrect conclusions if $\Delta_{i j}(T \sim$ $0) \sim \Delta_{i j}^{g}$, because $\Delta_{i j}(T)$ are roughly constant at the low temperatures relevant for compact star evolution. The exact condition for gapless quasiparticle excitations presented here [Eq. (16)], which is the main result of this paper, is a safe alternative to the approximative results. Moreover, Fig. 1 is an accurate qualitative picture of the prerequisites for gapless color-antitriplet excitations in NJL models.

\section{ACKNOWLEDGMENTS}

We thank D. Blaschke, M. Buballa, S. Fredriksson, and H. Grigorian for useful discussions and suggestions. A. M. Öztaş received support from Hacettepe University Research Fund, grant no. 0202602 001. F. Sandin acknowledges support from the Swedish National Graduate School of Space Technology and thanks D. Blaschke and the organizers of the Helmholtz International Summer School, Dubna, for partial support and their hospitality.
[1] B. C. Barrois, Nucl. Phys. B129, 390 (1977).

[2] D. Bailin and A. Love, Phys. Rep. 107, 325 (1984).

[3] M. G. Alford, K. Rajagopal, and F. Wilczek, Phys. Lett. B422, 247 (1998).

[4] R. Rapp, T. Schäfer, E. V. Shuryak, and M. Velkovsky, Phys. Rev. Lett. 81, 53 (1998).

[5] I. Shovkovy and M. Huang, Phys. Lett. B564, 205 (2003).

[6] M. Huang and I. Shovkovy, Nucl. Phys. A729, 835 (2003).

[7] M. Alford, C. Kouvaris, and K. Rajagopal, Phys. Rev. Lett. 92, 222001 (2004).

[8] M. Alford, C. Kouvaris, and K. Rajagopal, Phys. Rev. D 71, 054009 (2005).

[9] M. Alford, P. Jotwani, C. Kouvaris, J. Kundu, and K. Rajagopal, Phys. Rev. D 71, 114011 (2005).

[10] M. Huang and I. A. Shovkovy, Phys. Rev. D 70, 094030 (2004).
[11] M. Huang and I. A. Shovkovy, Phys. Rev. D 70, 051501(R) (2004).

[12] R. Casalbuoni, R. Gatto, M. Mannarelli, G. Nardulli, and M. Ruggieri, Phys. Lett. B605, 362 (2005).

[13] I. Giannakis and H.-C. Ren, Phys. Lett. B611, 137 (2005).

[14] K. Fukushima, Phys. Rev. D 72, 074002 (2005).

[15] H. Abuki and T. Kunihiro, Nucl. Phys. A768 118 (2006); hep$\mathrm{ph} / 0509172$.

[16] S. B. Rüster, V. Werth, M. Buballa, I. A. Shovkovy, and D. H. Rischke, Phys. Rev. D 72, 034004 (2005).

[17] D. Blaschke, S. Fredriksson, H. Grigorian, A. M. Öztaş, and F. Sandin, Phys. Rev. D 72, 065020 (2005).

[18] C. Kettner, F. Weber, M. K. Weigel, and N. K. Glendenning, Phys. Rev. D 51, 1440 (1995).

[19] M. Buballa and I. A. Shovkovy, Phys. Rev. D 72, 097501 (2005); hep-ph/0508197.

[20] M. Buballa and I. A. Shovkovy, Phys. Rev. D 72, 097501 (2005). 